\title{
A comparative study of anxiety and depression in patients with bronchial asthma, chronic obstructive pulmonary disease and tuberculosis in a general hospital of chest diseases Georgios Moussas*1, Athanasios Tselebis², Athanasios Karkanias², Dimitra Stamouli2 ${ }^{2}$ Ioannis Iliass ${ }^{3}$, Dionisios Bratis ${ }^{2}$ and Kalliopi Vassila- Demi $^{2}$
}

Address: ${ }^{1}$ Attikon General Hospital, Second Psychiatric Department, Medical School, University of Athens, Greece, ${ }^{2}$ Sotiria General Hospital of Chest Diseases, Psychiatric Department, Athens, Greece and ${ }^{3}$ Elena Venizelou Hospital, Athens, Greece

Email: Georgios Moussas* - gmpsuoa@gmail.com ; Athanasios Tselebis - atselebis@yahoo.gr; Athanasios Karkanias - apkarkanias@gmail.com; Dimitra Stamouli - demistam@otenet.gr; Ioannis Ilias - iiliasmd@yahoo.com; Dionisios Bratis - dionbratis@yahoo.gr; Kalliopi VassilaDemi - demkap@otenet.gr

* Corresponding author

\section{Published: 21 May 2008}

Annals of General Psychiatry 2008, 7:7 doi:10.1186/1744-859X-7-7
Received: 16 March 2007

Accepted: 21 May 2008

This article is available from: http://www.annals-general-psychiatry.com/content/7///7

(c) 2008 Moussas et al; licensee BioMed Central Ltd.

This is an Open Access article distributed under the terms of the Creative Commons Attribution License (http://creativecommons.org/licenses/by/2.0), which permits unrestricted use, distribution, and reproduction in any medium, provided the original work is properly cited.

\begin{abstract}
Background: Depression necessitating assistance from health professionals has a lifetime prevalence of $10 \%$. Chronic disease increases comorbidity with mood and/or anxiety disorders. Patients with chronic pulmonary disease present with severely impaired functionality, chronic somatic and psychogenic pain, require frequent hospitalizations and have a dependency upon medical and nursing personnel. In the present study we assessed anxiety and depression in patients hospitalized for pulmonary disease in a pulmonary disease hospital.

Methods: We assessed anxiety, using the Spielberger state-trait anxiety scale, and depression, using the Beck Depression Inventory, in 132 patients with pulmonary disease.

Results: A total of $49.2 \%$ of the sample had moderate or severe depression and $26.5 \%$ had anxiety. Women had higher depression and anxiety scores than men ( $t$ test, $p<0.05)$. Depression was positively correlated with anxiety, age and time from diagnosis. Anxiety was positively correlated with depression and time from diagnosis (Pearson $r=0.62$ and 0.29, $p<0.01$ ). Patients with chronic obstructive pulmonary disease and bronchial asthma had higher depression scores than patients with tuberculosis ( $t$ test, $p<0.01$ ).
\end{abstract}

Conclusion: Depression and anxiety are very prevalent in patients with pulmonary disease, especially chronic disease. This may be a very important negative factor in patients' adaptation to the chronic course of their disease.

\section{Background}

Among psychiatric diseases, depression, necessitating assistance from health professionals, has a lifetime preva- lence of $10 \%$ [1]. Furthermore, in the general population, depression has a point prevalence of $2.3 \%$ to $4.9 \%$. Up to $80 \%$ of patients with depression are either treated by non- 
mental health professionals or receive no treatment at all [2]. Chronic disease increases comorbidity with mood and/or anxiety disorders. Usually, the more serious the somatic disease is, the more probable it will be accompanied by mood and/or anxiety symptoms of variable severity. Failure to manage such mental health problems increases the patients' probability of suffering from complications, even lethal. The lifetime prevalence of mood disorder in patients with chronic disease is $8.9 \%$ to $12.9 \%$, with a 6 -month prevalence of $5.8 \%$ to $9.4 \%$. [3,4].

According to findings from worldwide research, $20 \%$ of patients with somatic disease suffer from major depression [4]. In relevant studies in Greece, $28.1 \%$ of patients hospitalized in general medical or surgical hospital wards had depression $[5,6]$.

In patients with pulmonary disease in particular, functionality may be severely impaired due to chronic psychogenic and somatic pain, frequent hospital admissions and dependency from medical and nursing personnel. The observed higher prevalence of depression and anxiety in patients with chronic pulmonary disease - compared to other chronic diseases - may be explained within this context. Despite the fact that clinical experience accepts high comorbidity in pulmonary patients, studies assessing and comparing anxiety and depression levels among patients with different pulmonary diseases are lacking in the Greek literature. In the present study we assessed anxiety and depressive symptoms in patients hospitalized in pulmonary clinics with bronchial asthma (BA), chronic obstructive pulmonary disease (COPD) or tuberculosis (TB).

\section{Methods}

Depression was assessed with the Beck Depression Inventory (BDI), which is widely used, and has been standardized and used in the Greek population previously [7-9]. The BDI, one of the most popular depression rating scales, includes 21 items graded from 0 to 3 . The inner coherence reliability is high and the re-test reliability ranges from 0.48 to 0.86 for clinical groups and 0.60 to 0.90 for nonclinical population. Its validity in relation to an external criterion for depression, such as clinical diagnosis, is considered to be satisfactory [7]. Anxiety was assessed with the Spielberger state-trait anxiety scale, one of the wellknown and broadly used anxiety rating scales. The scale consists of 40 items, each one graded from 1 to 4 . The scale differentiates anxiety to (a) anxiety caused by a specific condition (state subscale), and (b) anxiety as a more permanent characteristic of the personality (trait subscale). This second (trait) subscale was used in our study protocol. The scale is considered as having a high inner coherence reliability and validity compared to clinical diagnosis [10-12].

\section{Sample}

The sample included 140 hospitalized patients, of which 8 subjects refused to participate and were therefore excluded. The study included 132 patients $(78$ men and 54 women) in the pulmonary departments of our hospital. Of them, 42 were diagnosed with BA, 60 with COPD and 30 with TB. They were considered for enrollment over a 2-month period. All the participants were informed and gave their formal consent.

The subjects replied to the questionnaires in the presence of psychologists and/or psychiatrists familiarized with such tests. We assessed age, gender, years of education, duration of illness and diagnosis for hospitalization. Student's t test was used to assess differences in anxiety or depression between genders and among BA, COPD or TB patients. Pearson's correlation was used to assess the impact of anxiety on depression (and vice versa) as well as the impact of age or time from diagnosis on anxiety or depression. Logistic regression was used to assess the presence of depression (i.e. BDI scores $>13$ ) as a function of gender, age, time from diagnosis and anxiety. Descriptive statistics are given as mean \pm standard deviation (SD).

\section{Results}

The mean age of the sample was $54.08 \pm 16.60$ years and mean time from diagnosis was $8.78 \pm 9.14$ years. Men were older ( $57.44 \pm 15.16$ years) than women (49.22 \pm 17.50 years, two-tailed t test, $\mathrm{p}<0.05)$. There was no difference in the duration from diagnosis (two-tailed t test, $\mathrm{p}$ $=0.56$ ). Women had higher anxiety and depression scores than men (two-tailed t test, $\mathrm{p}<0.05$ ); $49.2 \%$ of the sample had moderate to severe depression, and $44.0 \%$ of men had depression symptoms compared to $55.6 \%$ of women, whilst $21.8 \%$ of men had anxiety symptoms compared to $33.3 \%$ of women (Table 1).

Depressive symptomatology was positively correlated with anxiety (Pearson $r=0.62, p<0.01)$, age (Pearson $r=$ $0.20, p<0.05$ ) and time from diagnosis (Pearson $r=0.39$, $p<0.01)$. The correlation of depression and age persisted when time from diagnosis was used as a control variable (partial correlation two-tailed $\mathrm{p}<0.05$ ). Anxiety was positively correlated with duration of illness (Pearson $\mathrm{r}=$ $0.29, \mathrm{p}<0.01)$. When depression was used as a control variable, the correlation of anxiety with time from diagnosis was not maintained (partial correlation $r=0.12, p>$ 0.05). Patients with COPD had the higher depression scores, followed by patients with BA, whereas patients with TB had the lowest depression scores. Anxiety was higher in patients with COPD compared to patients with TB. Patients with COPD were older and had more years of illness compared to those with BA and TB ( $t$ test $\mathrm{p}<0.05)$. $\mathrm{BA}$ patients were ill for a longer time compared to TB patients ( $\mathrm{t}$ test $\mathrm{p}<0.05)$ (Table 2 ). 
Table I: Patients studied by gender

\begin{tabular}{cccccc}
\hline Gender & & Age & Time from diagnosis (Years) & Anxiety & Depression \\
\hline Men $(n=78)$ & Mean & 57.45 & 9.18 & 42.44 & 12.71 \\
Women $(n=54)$ & SD & 16.16 & 10.22 & 9.87 & 8.09 \\
& Mean & 49.22 & 8.30 & 46.20 & 15.50 \\
Total $(n=132)$ & SD & 17.05 & 7.76 & 8.13 & 8.14 \\
& Mean & 54.08 & 9.18 & 43.98 & 13.85 \\
& SD & 16.60 & & 10.11 & 8.20 \\
\hline
\end{tabular}

We also examined depression in relation to anxiety, years of illness, gender and age by logistic regression. Logistic regression showed that anxiety seems to be the major determinant for depression $(\mathrm{p}<0.001)$ (Table 3$)$.

\section{Discussion}

The present study confirms that COPD patients are the group with the higher and more severe depression comorbidity. Depression in patients with chronic respiratory diseases coexists with anxiety and is related to the chronicity of the disease in our study, which has a negative effect on quality of life [13].

In recent years there has been a growing interest in the relationship between chronic pain and depression [14]. Chronic respiratory diseases such as COPD and BA entail serious subjective difficulties, chronic psychogenic and somatic pain, frequent hospital admissions, hospital dependency and dependency on oxygen. This metaphorically and literally suffocating disease status may explain the high percentage of depression in patients with COPD and BA in the study, which was higher than the percentage reported in studies performed with in-patients of general hospitals [5]. Furthermore, this difference is verified by studies performed with patients with respiratory failure, with depression being observed in $30 \%$ of patients with moderate failure and in $50 \%$ in patients with severe obstructive pulmonary disease $[15,16]$.

Anxiety and depression are very prevalent even in patients with moderate COPD (categorized as such by respiratory symptoms evaluation and functional tests using medical criteria) [17]. Chronic disease and comorbidity with anxiety and depression apparently leads to increased use of health services, approximately twice as often than in patients with no psychological burden [18]. Psychosocial stressors, such as death of a spouse or divorce, are closely related to relapses and aggravations of respiratory disease, especially in men, pointing to a link between psychological factors and chronic pulmonary disease [19]. Patients with COPD cannot cope adequately with everyday needs. This inadequacy may lead to heightened anxiety and depression, which in turn may worsen the everyday inadequacy. It has been reported that this is (probably) a factor that leads BA and COPD patients to frequent hospital admissions and even intensive care unit hospitalizations [20].

It is accepted that current psychiatric practice has valid ways to diagnose depression, implementing different diagnostic criteria and taxonomic systems such as the Diagnostic and Statistic Manual IV (DSM-IV) and International Classification of Disorders (ICD-10). The pathogenesis of depression is becoming better understood and therapy has a very high success rate. This progress sets the necessity for a more successful detection of all forms of depression, especially in chronic somatic patients and in the elderly; a group in which depression often escapes diagnosis, although in the elderly COPD is an important cause of morbidity, disability and mortality [21]. As mentioned previously, COPD and BA are chronic diseases with severe subjective difficulties, dependency on medical

Table 2: Patients studied by disease

\begin{tabular}{|c|c|c|c|c|c|}
\hline Gender & & Age & Time from diagnosis (Years) & Anxiety & Depression \\
\hline \multirow[t]{2}{*}{ Bronchial asthma $(n=42)$} & Mean & 43.33 & 9.24 & 43.67 & $|4.3|$ \\
\hline & SD & 14.28 & 6.90 & 9.96 & 7.45 \\
\hline \multirow[t]{2}{*}{$\operatorname{COPD}(n=60)$} & Mean & 66.03 & 13.46 & 45.87 & 15.48 \\
\hline & SD & 9.61 & 10.57 & 10.36 & 8.42 \\
\hline \multirow[t]{2}{*}{ Tuberculosis $(n=30)$} & Mean & 45.23 & 2.69 & 40.67 & 9.93 \\
\hline & SD & 14.99 & 6.51 & 9.19 & 7.71 \\
\hline \multirow[t]{2}{*}{ Total $(n=132)$} & Mean & 54.08 & 8.78 & 43.98 & 13.85 \\
\hline & SD & 16.60 & 9.15 & 10.11 & 8.20 \\
\hline
\end{tabular}

COPD, chronic obstructive pulmonary disease. 
Table 3: Pearson correlation scores

\begin{tabular}{|c|c|c|c|c|c|}
\hline & & Depression & Anxiety & Age & Time from diagnosis (Years) \\
\hline \multirow[t]{2}{*}{ Depression } & Pearson correlation coefficient $(n=132)$ & 1.00 & 0.62 & 0.20 & 0.39 \\
\hline & Two-tailed $p$ value & - & $<0.01$ & $<0.05$ & $<0.01$ \\
\hline \multirow[t]{2}{*}{ Anxiety } & Pearson correlation coefficient $(n=132)$ & 0.62 & 1.00 & 0.13 & 0.29 \\
\hline & Two-tailed $p$ value & $<0.01$ & - & 0.14 & $<0.01$ \\
\hline \multirow[t]{2}{*}{ Age } & Pearson correlation coefficient $(n=132)$ & 0.20 & 0.13 & 1.00 & 0.34 \\
\hline & Two-tailed $p$ value & $<0.05$ & 0.14 & - & $<0.01$ \\
\hline \multirow[t]{2}{*}{ Time from diagnosis } & Pearson correlation coefficient $(n=107)$ & 0.39 & 0.29 & 0.34 & 1.00 \\
\hline & Two-tailed $p$ value & $<0.01$ & $<0.01$ & $<0.01$ & - \\
\hline
\end{tabular}

and nursing personnel and dependency on oxygen. In these diseases depression ranges from $30 \%$ in moderate up to $50 \%$ in severe forms of COPD [15], a fact also verified in our study. Depression may be a very important negative factor to treatment adherence for patients with somatic disease. Additionally, it may hinder adaptation to chronic disease conditions and it is known that adaptation is a crucial survival factor in chronic diseases [22].

\section{Conclusion}

Patients suffering from BA and COPD have a significantly higher rate of anxiety and depression compared to the general population. A probable cause is the chronicity and severity of pulmonary disease. Detection and management of these mental disorders may ameliorate prognosis of the pulmonary disease and improve adaptation and quality of life of these patients.

\section{Competing interests}

The authors declare that they have no competing interests.

\section{References}

I. Wells KB, Golding J, Burnan MA: Psychiatric disorder in a sample of the general population with and without chronic medical conditions. Am J Psychiatry 1998, I 45:976-979.

2. Regier DA, Goldberg ID, Tance CH: The de facto U.S. mental health systems. Arch Gen Psychiatry 1978, 35:685-693.

3. Cassen EH: Depression and anxiety secondary to medical illness. Psychiatr Clin North Am 1990, I3:597-6I 2.

4. Cassem NH, Bernstein JG: Depressed patients. In Massachusetts General Hospital Handbook of General Hospital Psychiatry 5th edition. Edited by: Stern TA, Fricchione GL, Cassen WH, Jellinek MS, Rosenbanm JF. Philadelphia, PA: Mosby/Elsevier; 2004:25-68.

5. Lykouras $E$, loannidis $H$, Voulgaris $A$ : Depression in general hospital patients: preliminary results. Arch Hell Med 1987, 4:287-289.

6. Giannitsi S, Liakos A: Preliminary observations after implementation of two years of liaison psychiatry service in a general hospital. Encephalos 1985, 22:138.

7. Beck AT, Steer RA: Manual for the Revised Beck Depression Inventory San Antonio, TX: Psychological Corporation; 1987.

8. Donias S, Demertzis I: Validation of the Beck depression inventory. In Proceedings of the 10th Hellenic Congress of Neurology and Psychiatry: 1983 Edited by: Varfis G. Thessaloniki, Greece: University Studio Press; 1983:486-492.

9. Tselebis A, Moulou A, llias I: Burnout versus depression and sense of coherence: A study in Greek nursing staff. Nursing Health Sci 200I, 3:69-7I.

10. Spielberger GD, Gorush RL, Lusshene RE: The State-Trait Anxiety Inventory Palo Alto, CA: Consulting Psychologists Press; 1970.
II. Liakos A, Giannitsi S: The validity of the Greek modification of the Spielberger anxiety scale. Encephalos 1984, 2 I:7I-76.

12. Tselebis A, Papaleftheris E, Balis E, Theotoka I, Ilias I: Smoking related to anxiety and depression in Greek medical staff. Psycholog Rep 2003, 92:529-532.

13. Anderson KL: The effect of chronic obstructive pulmonary disease on quality of life. Res Nurs Health 1995, 18:547-556.

14. Campell LC, Claw DJ, Keefe FJ: Persistent pain and depression: a biopsychosocial pespective. Biol Psychiatry 2003, 54:399-409.

15. Kunik ME, Roundy K, Veazey C, Souchek J, Richardson P, Wray NP, Stanley MA: Surprisingly high prevalence of anxiety and depression in chronic breathing disorders. Chest 2005, 127:|205-12|1.

16. Light RW, Merrill EJ, Despars JA, Gordon GH, Mutalipassi LR: Prevalence of depression and anxiety in patients with COPD. Relationship to fuctional capacity. Chest 1985, 87:35-38.

17. Di Marco F, Verga M, Reggente M, Maria Casanova F, Santus P, Blasi $F$, Allegra L, Centanni S: Anxiety and depression in COPD patients: the roles of gender and disease severity. Respir Med 2006, 100:1767-1774.

18. Gudmundsson G, Gislason T, Janson C, Lindberg E, Suppli Ulrik C, Brondum E, Nieminen MM, Aine T, Hallin R, Bakke P: Depression, anxiety and health status after hospitalization for COPD: a multicentre study in the Nordic countries. Respir Med 2006, 100:87-93.

19. Cicutto LC, Brooks D: Self-care approaches to managing chronic obstructive pulmonary disease: A provincial survey. Respir Med 2006, 100:1540-1546.

20. Miller KE: Do anxiety and depression impact patients with COPD? Am Family Physic 2003, 67:1327.

21. Yohannes AM, Baldwin RC, Connolly MJ: Depression and anxiety in elderly patients with chronic obstructive pulmonary disease. Age Ageing 2006, 35:457-459.

22. Vanderpool M: Resilience: a missing link in our understanding of survival. Harv Rev Psychiatr 2002, 10:302-306.

Publish with Bio Med Central and every scientist can read your work free of charge

"BioMed Central will be the most significant development for disseminating the results of biomedical research in our lifetime. "

Sir Paul Nurse, Cancer Research UK

Your research papers will be:

- available free of charge to the entire biomedical community

- peer reviewed and published immediately upon acceptance

- cited in PubMed and archived on PubMed Central

- yours - you keep the copyright 